\title{
A Hybrid Image Denoising Method
}

\author{
Hari Om \\ Department of Computer Science \& Engineering, \\ Indian School of Mines, Dhanbad, \\ Jharkand-826004, India
}

\author{
Mantosh Biswas \\ Department of Computer Science \& Engineering, \\ Indian School of Mines, Dhanbad, \\ Jharkand-826004, India
}

\begin{abstract}
In this paper, a hybrid image denoising method that is based on locally adaptive window-based maximum likelihood (LAWML) and NeighShrink. The LAWML is doubly stochastic process models which denoise an image by exploiting the dependency of local wavelet coefficients within each scale. The LAWML needs a global optimal neighboring window. The NeighShrink thresholding scheme uses the immediate neighboring coefficients based on block thresholding. It uses a suboptimal universal threshold and identical neighbouring window size in all wavelet subbands. The NeighShrink and LAWML always produce an over-smoothed image like the Weiner filter in which many of the detail coefficients are lost during threshold evaluation. This proposed method overcomes these disadvantages and, as a result, it provides significant improvement in visual quality i.e. Peak-to-Signal Noise Ratio (PSNR) of a noisy image.
\end{abstract}

\section{Keywords}

Image Denoising, Thresholding, LAWML, NeighShrink, Peakto-Signal Noise Ratio (PSNR)

\section{INTRODUCTION}

Developing a method that is capable of suppressing the additive white noise completely from a noisy image without removing the image details is still a challenging task. Some of the following vital criteria's for designing a denoising technique are: the additive noise in smooth regions should completely be removed, the edges should not be blurred/sharpened, the texture details should be maintained and the overall contrast should be preserved, and finally the additional artifacts should not appear in the restored image. It is however very difficult to develop a denoising method that matches all these criteria. An efficient signal denoising algorithm should remove the noise from the signal while preserving the useful information in signal as much as possible. In last decade and so, the non-wavelet based image denoising methods such as Median filter, Weiner filtering have been discussed that remove the noise but at the cost of blurring images which in turn makes the edges of blurring images which in turn makes the edges in pictures/images invisible [1]. These methods generally work well only for a smooth signal that is corrupted by Gaussian noise and require the information about spectra of noise and original signal. The wavelet based analysis has been demonstrated to be one of the powerful methods over non-wavelet methods for performing image noise reduction [23]. The motivation for using a wavelet based method is that it is good for energy compaction since the small and large coefficients are more likely due to the noise and important signal features, respectively. Recently, there have been discussed many denoising methods that take local characteristics into account [4-10], [15-19]. The most commonly used approaches for denoising are: hard and soft thresholding [4]. Due to their effectiveness and simplicity, these approaches are frequently used in literature. The main idea behind thresholding based methods is to subtract the threshold value from all the coefficients that are larger than the threshold and set all other coefficients to zero. The VisuShrink uses wellknown threshold that is known as the universal threshold [4-5]. Cai and Silverman have discussed two thresholding schemes: NeighCoeff and NeighBlock. These schemes take immediate neighboring coefficients into account [6]. They have shown experimentally that the thresholding of neighboring coefficients is better than the traditional term-by-term wavelet thresholding. Chen and Bui have developed the NeighShrink method which follows VisuShrink threshold [7-8]. Mahqak et al. have discussed an efficient denoising method that is known as locally adaptive window-based maximum likelihood (LAWML) method [9-10]. In this method, the wavelet coefficients satisfy the Gaussian distribution with zero-mean. The variance of the wavelet coefficients is obtained by maximum likelihood estimation in square neighborhood and the denoised wavelet coefficients are obtained by using minimum mean squared error-like (MMSE-like) estimation. This proposed method deals with 'local' spatially adaptive statistical model based on LAWML and NeighShrink with new shrinkage factor. It overcomes the limitations of LAWML and NeighShrink methods and performs better than these methods. The structure of the paper is organized in five sections. In section 2, we discuss about the wavelet analysis and section 3 reviews the related work. The proposed method is presented in section 4. In section 5, simulation results are presented and discussions of the proposed method. Finally, the conclusion is given in section 6.

\section{WAVELET ANALYSIS}

Wavelet-based tools are now indispensable in many areas of modern statistics, especially in regression, density and function estimation, factor analysis, modeling and forecasting of time series, functional data analysis and classification, with ranges of application areas in science and engineering [11]. A wavelet transform can help localizing a signal well in both time and scale (frequency). The wavelet transforms adaptively distribute the time-frequency precision by their innate nature [12]. In a wavelet transform, the decomposition of a signal with a family of real orthonormal bases $\psi_{m, n}(x)$ is obtained through translation and dilation of a kernel function $\psi(\mathrm{x})$, known as the mother wavelet [13] i.e.

$$
\psi_{m, n}(x)=2^{(-m / 2)} \psi\left(2^{-m} x-n\right)
$$

(1) 
where, $\mathrm{m}$ and $\mathrm{n}$ are integers.

The wavelet coefficients of a signal $f(x)$ obey the orthonormal property and can be easily computed as follows:

$$
C_{m, n}=\int_{-\infty}^{\infty} f(x) \psi_{m, n}(x) d x
$$

and the synthesis formula is given by:

$$
F(x)=\sum_{m, n} C_{m, n} \psi_{m, n}(x)
$$

To construct the mother wavelet, $\psi(\mathrm{x})$, a scaling function $\phi(x)$ is needed that satisfies two scale difference equation as given below:

$$
\phi(x)=\sqrt{2} \sum_{k} h(k) \phi(2 x-k)
$$

The wavelet $\psi(\mathrm{x})$ is related to the scaling function as given below:

$$
\psi(x)=\sqrt{2} \sum_{k} g(k) \phi(2 x-k)
$$

where, $\mathrm{g}(\mathrm{k})=(-1)^{\mathrm{k}} \mathrm{h}(1-\mathrm{k})$

The $\mathrm{J}^{\text {th }}$ level wavelet decomposition can be written as:

$$
F(x)=\sum C_{o, k} \phi_{o, k}(x)=\sum_{k}^{K}\left(C_{j+1, k} \phi_{j+1, k}(x)+\sum_{j=0}^{J} d_{j+1, k} \psi_{j+1, k}(x)\right)
$$

The coefficients $\mathrm{C}_{\mathrm{o}, \mathrm{k}}$ are given. These are indeed samples of original signal. The coefficients $C_{j+1}$ and $d_{j+1, k}$ are at $(j+1)^{\text {th }}$ scale that are related to the coefficients $C_{j, k}$ at ${ }^{\text {th }}$ scale via

$$
\begin{aligned}
& C_{j+1, n}=\sum_{k} C_{j \cdot k} h(k-2 n) \\
& d_{j+1, n}=\sum_{k} C_{j . k} g(k-2 n) \quad 0 \leq \mathrm{j} \leq \mathrm{J}
\end{aligned}
$$

Thus, it provides recursive algorithm for wavelet decomposition through $\mathrm{h}(\mathrm{k})$ and $\mathrm{g}(\mathrm{k})$ and the final outputs include a set of $\mathrm{J}^{\text {th }}$ level wavelet coefficients $\mathrm{d}_{\mathrm{j}, \mathrm{n}}, 1 \leq \mathrm{j} \leq \mathrm{J}$ and the coefficients $\mathrm{C}_{\mathrm{j}, \mathrm{n}}$ for low resolution component $\phi_{j, k}(x)$. By using a similar approach, we can derive recursive algorithm for synthesis based on its wavelet coefficients $\mathrm{d}_{\mathrm{j}, \mathrm{n}}, 1 \leq \mathrm{j} \leq \mathrm{J}$ and $\mathrm{C}_{\mathrm{j}, \mathrm{n}}$

$$
C_{j, k}=\sum_{n} C_{j+1 . n} h(k-2 n)+\sum_{n} d_{j+1 . n} g(k-2 n)
$$

It is convenient to view the decomposition as passing the signal through a pair of filters $\mathrm{H}$ and $\mathrm{G}$ with impulse responses $\mathrm{h}(\mathrm{n})$ and $g(n)$, respectively, and down-sampling the filtered signals by two, where the functions $h(n)$ and $g(n)$ are even i.e.

$$
\mathrm{h}(\mathrm{n})=\mathrm{h}(-\mathrm{n}) \& \mathrm{~g}(\mathrm{n})=\mathrm{g}(-\mathrm{n})
$$

The pair of filters $\mathrm{H}$ and $\mathrm{G}$ corresponds to the half-band low pass and high pass filters, respectively, and is also called the quadrature mirror filters in the signal processing terminology as it is shown in Fig. 1 [14]. The reconstruction procedure is implemented by up-sampling the sub signals $C_{j+1}$ and $d_{j+1}$ (inserting zero between the neighboring samples) and filtering with $h(n)$ and $g(n)$, respectively, and finally, adding these two filtered signals together. Usually, the signal decomposition scheme is performed recursively to the output of low pass filter h. It leads to conventional wavelet transform that is also called pyramid structured wavelet decomposition.

The wavelet equation produces different wavelet families like Daubechies, Symlet, Haar, coiflets, etc. They are also classified into a family by the number of vanishing moments. Within each family of wavelets, there are wavelet subclasses distinguished by the number of coefficients and the level of iterations. The wavelet decomposition of an image is done as follows. In first level of decomposition, the image is split into four subbands, namely HH, HL, LH and LL subbands as shown in Fig. 2. The HH and HL subbands give diagonal and horizontal feature details of the image, respectively, while the LH subband represents the vertical structures. The LL subband is the low resolution residual consisting of low frequency components and it is the only subband which is further split at higher levels of composition.

\begin{tabular}{|c|c|c|}
\hline $\mathrm{LL}_{2}$ & $\mathrm{HL}_{2}$ & \\
$\mathrm{LH}_{2}$ & $\mathrm{HH}_{2}$ & \\
\hline \multicolumn{2}{|c|}{$\mathrm{HL}_{1}$} \\
\hline \multicolumn{2}{|c|}{$\mathrm{LH}_{1}$} & $\mathrm{HH}_{1}$ \\
\end{tabular}

Fig. 2: 2D-DWT with 2-Level decomposition

\section{RELATED WORK}

Wiener filtering is a non-wavelet method, which can remove noise while protecting the edges in an image [1]. However, this method performs poorly in the presence of signal dependent noise. The Wiener filter in Fourier domain is given as follows:

$$
G(i, j)=\frac{H^{*}(i, j)}{|H(i, j)|^{2}+\frac{P_{n}(i, j)}{P_{s}(i, j)}}
$$

where, $\operatorname{MxM}(1 \leq i, j \leq M)$ is the number of image pixels, $\mathrm{H}(\mathrm{i}, \mathrm{j})$ denotes degradation function, $\mathrm{H}^{*}(\mathrm{i}, \mathrm{j})$ its conjugate; $\mathrm{P}_{\mathrm{n}}(\mathrm{i}, \mathrm{j}), \mathrm{P}_{\mathrm{s}}(\mathrm{i}$, $\mathrm{j}$ ), and $\frac{P_{n}(i, j)}{P_{s}(i, j)}$ denote power spectral density of noise, nondegraded image, and reciprocal of the signal-to-noise ratio, respectively. To overcome the above weaknesses of the Wiener filtering, Donoho and Johnstone have discussed a wavelet based

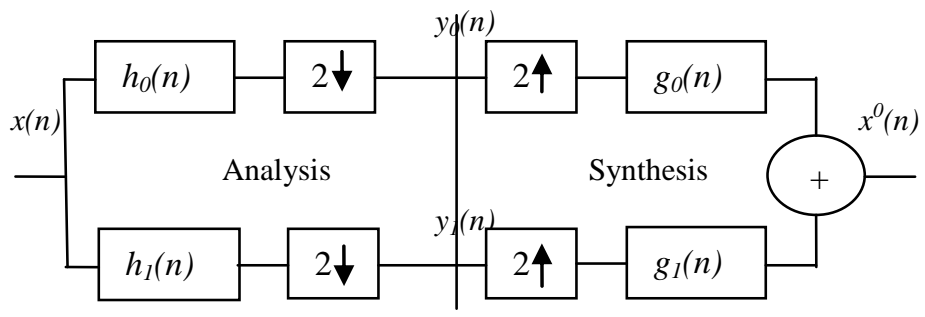

Fig. 1: Wavelet decomposition (Analysis) and reconstruction (Synthesis) filter model

denoising scheme [4-5]. They have given a mechanism to find the threshold value, which is known as VisuShrink [4]. The VisuShrink threshold is evaluated by the following formulae:

$$
\mathrm{T}_{\mathrm{Visu}}=\sigma \sqrt{2 \log M}
$$


where, $\sigma^{2}$ is the noise variance that defines the median absolute deviation as given below:

$$
\sigma^{2}=\left[\frac{\text { median }\left|H H_{1}(i, j)\right|}{0.6745}\right]^{2}
$$

The VisuShrink has been found to yield an overly smoothed image since the estimate is derived under the constraint with high probability.

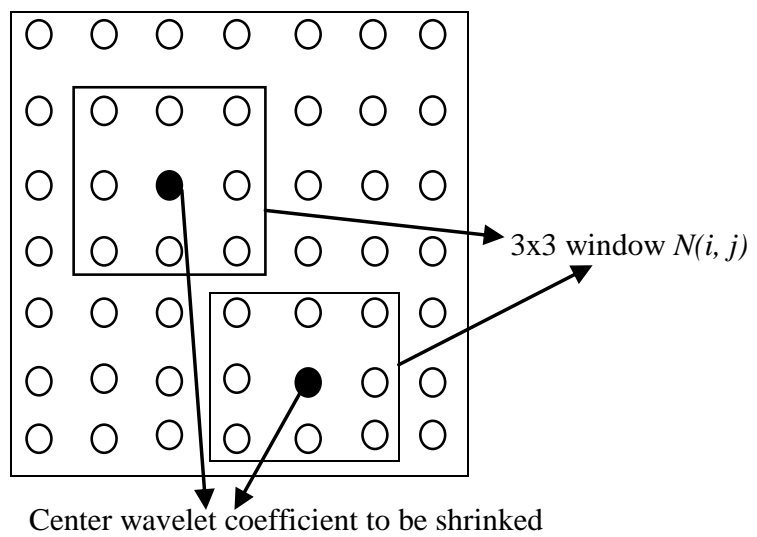

Fig. 3: Wavelet coefficients in neighborhood $3 \times 3$ window

Mahqak et al. have developed an image denoising method: LAWML, however, this method is not efficient because it does not determine a local optimal neighboring window size [9]. They obtain $\sigma_{i, j}^{2}$ the signal variance by using a locally adaptive window-based maximum likelihood (LAWML) estimation that gives reasonably good results and it is given by:

$$
\sigma_{i, j}^{2}=\max \left(0, \frac{1}{b} \sum_{S_{i, j} \in N(i, j)} S_{i, j}^{2}-\sigma_{n}^{2}\right)
$$

where, $N(i, j)$ represents a local window of size $\mathrm{L} \times \mathrm{L}$, having $b$ as the number of its coefficients. Here $L$ is a positive odd number, for example, the window size can be $3 \times 3,5 \times 5$ and so on. The $3 \times 3$ neighborhood window $N(i, j)$ is shown in Fig. 3, whose center is a wavelet coefficient to be thresholded.

Suppose that the wavelet coefficients satisfy the Gaussian distribution with zero-mean, the variance of wavelet coefficients can be computed by maximum likelihood estimation in square neighborhood. The denoised wavelet coefficient, denoted by $\hat{S}_{i, j}$, of the wavelet coefficients $S_{i, j}$ is given by:

$$
\hat{S}_{i, j}=\frac{\sigma_{i, j}^{2}}{\sigma_{i, j}^{2}+\sigma_{n}^{2}} S_{i, j}
$$

where, $\sigma_{i, j}^{2}$ is the signal variance for the wavelet coefficient $S_{i, j}$ that is assumed to be an independent Gaussian variable of the noisy coefficient.

The NeighShrink method [7-8] also incorporates neighboring coefficients in the thresholding process by considering a local window (square) of length L (a positive odd number), but it uses Universal threshold (refer Fig. 3). The coefficients of different subbands are thresholded

independently; however, the threshold and neighbouring window size are kept unchanged in all subbands. Let $\operatorname{Sum}_{i, j}^{2}$ denote the sum of square of the wavelet coefficients $S_{i, j}$ in the neighboring window $N(i, j)$ i.e.

$$
S u m_{i, j}^{2}=\sum_{S_{i, j} \in N(i, j)} S_{i, j}^{2}
$$

Now shrink the wavelet coefficients according to James-Stein (JS) rule that is defined below [7-8]:

$$
\hat{S}_{i, j}=S_{i, j}\left(\left(1-\frac{T_{V i s u}^{2}}{S u m_{i, j}^{2}}\right)_{+}\right)
$$

here, + sign at the end of the formula means to keep the positive value, and set it to zero when it is negative.

\section{PROPOSED METHOD}

Suppose $S$ is the noisy observed image of the original image R corrupted by noise $N$. The noise $\mathrm{N}$ is assumed to be an independent and identically distributed (i.i.d) white Gaussian noise with zero mean and finite variance $\sigma_{n}^{2}$. It can be written

$$
\mathrm{S}=\mathrm{R}+N
$$

Let $W(\cdot)$ and $W^{l}(\cdot)$ denote the forward and inverse wavelet transform operators [14] and $D(\cdot, T)$ denote the denoising operator with threshold $T$. Now apply $W($ ) to the noisy image $\mathrm{S}$, followed by $D(\cdot, T)$ and finally apply $W^{l}\left({ }^{\prime}\right)$ to the resultant. To carry out these steps, firstly we need to estimate the shrinkage factor that is discussed below.

\subsection{Parameter Estimation}

The estimation of shrinkage factor, $\beta_{i, j}$ that requires the threshold estimator value $\lambda$ is given below:

$$
\beta_{i, j}=\left(1-\left(\frac{n}{n+1}\right)\left(\frac{\lambda^{2}}{S u m_{i, j}^{2}}\right)\right)_{+}
$$

where, $n$, noise reduction factor, is a positive integer i.e., $0<\mathrm{n}$ $<\infty$. The choice of $n$ is independent to image, noise, subband, or scale. It has been observed that for high noise level, the higher value of $n$ gives good quality of image i.e. high PSNR. The threshold estimator $\lambda$ depends on the noise variance and size of subband and it is given as follows:

$$
\lambda=\sqrt{2 \sigma \log (\hat{M})}
$$

where, $\hat{M}=\mathrm{M} / 2^{l} ; 1 \leq l \leq \mathrm{J}$, here $\mathrm{J}$ represents the number of decomposition levels.

Now, shrink the wavelet coefficients according to the following expressions:

$$
\hat{S}_{i, j}=S_{i, j} \beta_{i, j}
$$

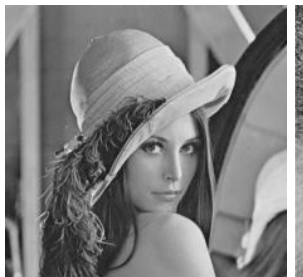

(a)

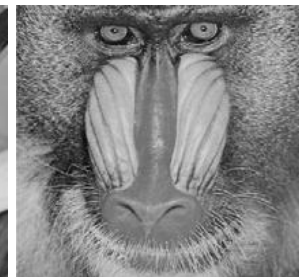

(b)

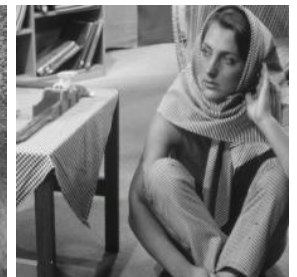

(c) 


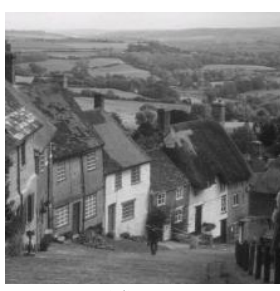

(d)

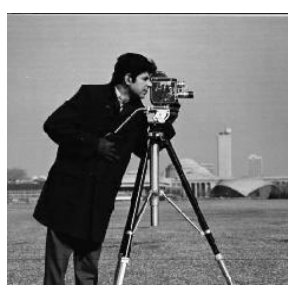

(e)
Fig. 4: Original test images of size $512 \times 512$ pixels: (a) Lena (b) Mandrill (c) Barbara (d) Goldhill (e) Cameraman

Once obtaining the shrinkage factor, apply the denoising procedure, which is discussed below. In this method, we use wavelet transform to obtain the wavelet coefficients of the image and then modify these coefficients. Finally, apply inverse wavelet transform to obtain an approximation of the original image.

\subsection{Denoising Procedure}

(i) Apply 2-D Wavelet Transformation $\mathrm{W}$ on the degraded image $\mathrm{S}$ to generate $\mathrm{J}$ decomposition levels.

(ii) For each subband except $\mathrm{LL}_{\mathrm{k}}$, for $1<k \leq\lfloor J / 2\rfloor$, apply (15) and, for $\lfloor J / 2\rfloor+1<k \leq J$, apply (21), to get the noiseless coefficients $\bar{R}$ from the noisy image $\mathrm{S}$.

(iii) Perform the inverse wavelet transformation $\mathrm{W}^{-1}$ to $\bar{R}$, modified coefficients, to obtain the denoised estimate image $\hat{S}$.

\section{EXPERIMENTAL RESULTS}

To test the superiority of this proposed method, the experimental study has been performed on the following images: Lena, Mandrill, Barbara, Goldhill, and Cameraman, each of size $512 \times 512$ (refer Fig. 4). These images are corrupted by the additive zero-mean Gaussian noise with different noise levels: $10,20,30,50,75$, and 100. In our experiments, we have used Symlet wavelet of length eight up to four decomposition levels is used and the square-shaped windows of sizes $3 \times 3$, $5 \times 5$, and $7 \times 7$ have been employed to find different estimations for $\sigma_{i, j}^{2}$ and $S u m_{i, j}^{2}$. The experimental results are evaluated for both objective and subjective quality of the images as these two parameters are widely used for statistical computations. The peak signal-to-noise ratio (PSNR) and visual quality of the restored images are considered for objective and subjective comparisons, respectively. In the proposed method, it is suggested to take the large values of $\mathrm{n}$ and the neighboring window size when the noise level is high.

It is observed that the proposed method outperforms the Weiner Filter in terms of PSNR for all window sizes: $3 \times 3,5 \times 5$, and $7 \times 7$, for all values of $n(n=1,2$, and 4$)$, and at all noise levels: 10, 20, 30, 50, 75, and 100 for all test images under consideration (refer Table 1 and Figs. 5(a)-(c)). Furthermore, our proposed method outperforms the NeighShrink and LAWML in terms of PSNR for window size 3x3, all values of $n$ ( $\mathrm{n}=1,2,4)$ and at all noise levels: 10, 20, 30, 50, 75, and 100 for all test images (refer Fig. 5(a)).
For window size $5 \times 5$ and NeighShrink, noise levels higher than 20 and any value of $\mathrm{n}$, this proposed method performs better than the NeighShrink for the Lena \& Goldhill images and for noise levels $10 \& 20$, it has no better performance. For Mandrill, Barbara, Cameraman images and noise level higher than 30 the performance of our method is better than the NeighShrink and for noise levels 10, $20 \& 30$, it is comparable (refer Fig. 5(b)).

In comparison to LAWML with Window size $5 \times 5$, the proposed method has no better results for Lena, Mandrill, Barbara, Cameraman images for any value of $\mathrm{n}$ and noise level 10 , but for noise level higher than 10 , it provides better results. For noise level 10 and $n=1$ in Goldhill image, it has comparable performance, but for noise level higher than 10 , it provides better results (refer Fig. 5(b))

For Window size $7 \times 7$ and NeighShrink, the proposed method gives better results than the NeighShrink for noise level 10 and for any value of $\mathrm{n}(=1,2$, and 4$)$ in all test images.

For $\mathrm{n}=1$, our results are no better for Lena, Barbara, Goldhill, and Cameraman when noise level is 20 or more. In Mandrill for $n=1$, our results are better for noise levels 50 and 75 , whereas our results are no better for noise levels: 20, 30, and 100 .

For $\mathrm{n}=2$ and Lena, and Mandrill images, our results are better for noise levels $50 \& 75$ and for noise levels 20, 30, and 100, they are comparable. For Barbara and Cameraman images, our results perform better for noise level 75 and, for noise levels 20,30, and 50, it is no better. For noise level 100, our results are better for Cameraman image and, for Barbara image, they are no better. For Goldhill image, our results are better for noise level 50 and, for noise levels 20, 30, $75 \& 100$, they are comparable.

For $\mathrm{n}=4$ and Lena, Mandrill \& Barbara images, our results are better for noise levels 50 or more and for noise levels $20 \&$ 30 , they are no better. For Goldhill image, our results are better for noise levels $50 \& 75$ and for noise levels $20,30 \& 100$, they are comparable. For Cameraman image, our method performs better for noise levels: $75 \& 100$ and, for noise levels $20,30 \&$ 50, they are no better (refer Fig. 5(c)).

In comparison to LAWML with window size $7 \times 7$, for $n=1$ and Lena images, our results are better than that of the LAWML for noise level 30, 50 and 75 and for noise levels 10, 20 and 100 they are comparable. For Cameraman images, our results are better than that of the LAWML for noise level 30 or more and for noise levels 10 and 20 they are comparable. For Mandrill \& Barbara images and noise levels 20, 30, 50, and 75, ours are better and for noise levels $10 \& 100$, they are comparable. For Goldhill image and noise levels 20, 30, and 50, ours are better and for noise levels $10,75 \& 100$, they are comparable.

For $n=2$ and Mandrill \& Goldhill images, our results are better for noise levels 20, 30, 50 and 75, and for noise levels 10 $\& 100$, they are comparable. For Barbara image, our results are better noise level 20 or more and comparable for noise level 10 . For Lena and Cameraman images, our method performs better for noise level is 30 or more and for noise levels $10 \& 20$, they are comparable. 
For $\mathrm{n}=4$ and Lena, Mandrill \& Cameraman images, our results are better for noise level 30 or more and for noise levels $10 \& 20$, they are comparable. For Barbara image, ours are better noise level 20 or more and for noise level 10, they are comparable. For Goldhill image, our method performs better for noise levels 20, 30, $50 \& 75$ and for noise levels 10 and 100, they are comparable (refer Fig. 5(c)).

We have shown the graphs for the denoised image of Lena in Fig. 5(a)-(c) for noise levels: 10, 20, 30, 50, 75, and 100 which gives a remarkable improvement over the Wiener filter, NeighShrink, and LAWML methods. Table 1 and Fig. 5(a) of suggest window of size $3 \times 3$. Furthermore, it is suggested to take the lower/higher value of $\mathrm{n}$ when the noise level is lower/higher, respectively, from the above discussion. Here, we have considered only Lena image for graph purpose. We have obtained similar types of PSNR curves for other images since the repetitive nature of results; we have not shown their graphs.

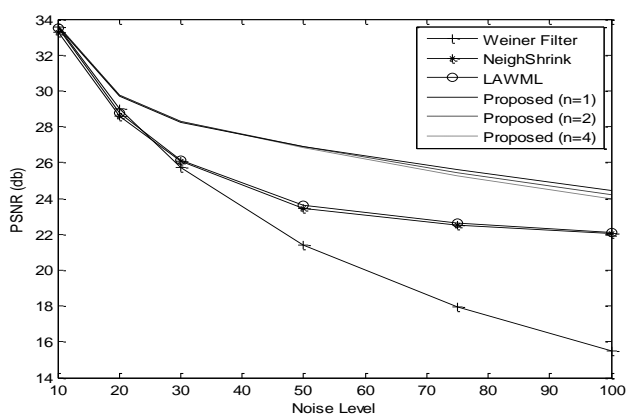

(a) Window size $3 \times 3$

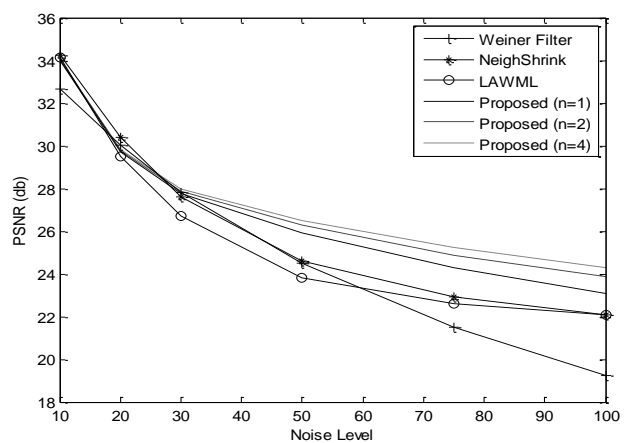

(b) Window size $5 \times 5$

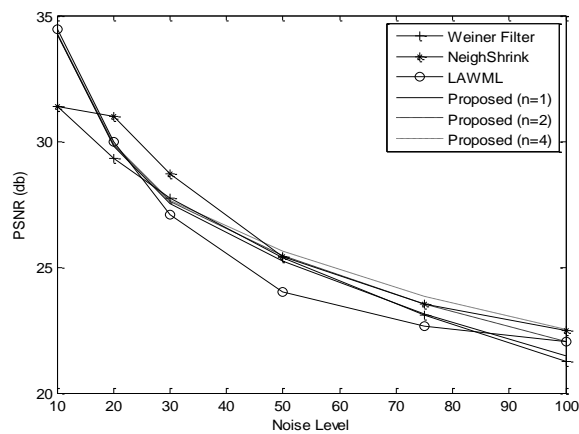

(c) Window size $7 \times 7$

Fig. 5: PSNR gain vs. Noise level of Proposed ( $n=1,2$ \& 4), Weiner Filter, NeighShrink, and LAWMAL methods for Lena with window size $3 \times 3$ in Fig. (a), $5 \times 5$ in Fig. (b) and $7 \times 7$ in Fig. (c)

\section{CONCLUSION}

In this paper, we have discussed a hybrid image denoising method based on LAWML and NeighShrink methods. This proposed method removes the noise from the noisy image significantly. It has either better performance than or comparable in terms of PSNR to the Weiner filter, NeighShrink, and LAWML. In future, we try to extend this method for multichannel /medical images, and video.

\section{ACKNOWLEDGMENTS}

Our sincere thanks goes to Prof. S. Chand for his invaluable comments and suggestions.

\section{REFERENCES}

[1] R. C. Gonzalez and R. E. Woods, Digital Image Processing, Prentice Hall, 2008.

[2] J. C. Xie, "Overview on Wavelet Image Denoising," Journal of Image and Graphics, Vol. 7(A), No. 3, 2002, pp. 209-217.

[3] A. G. Bruce and H. Y. Gao, "Understanding Wave Shrink: variance and bias estimation," Biometrika, Vol. 83, No. 4, 1996, pp.727-745.

[4] D. L. Donoho and I. M. Johnstone, "Ideal spatial adaptation via wavelet shrinkage," Biometrika, Vol. 81, No. 3, 1994, pp. 425- 455.

[5] D. L. Donoho, "De-Noising by Soft Thresholding," IEEE Trans. Information Theory, Vol. 41, No. 3, 1995, pp. 613627.

[6] T. T. Cai and H. H. Zhou, "A Data-Driven Block Thresholding Approach To Wavelet Estimation," Ann. Statist., Vol. 37, No. 2, 2009, pp. 569-595.

[7] G. Y. Chen and T. D. Bui, "Multiwavelets Denoising Using Neighboring Coefficients," IEEE Signal Processing Letters, Vol. 10, No. 7, 2003, pp. 211-214.

[8] G. Y. Chen, T. D. Bui and A. Krzyzak, "Image Denoising Using Neighbouring Wavelet Coefficients," ICASSP, 2004, pp. 917-920.

[9] M. K. Mihcak, I. Kozintsev, and K. Ramchandran, "Spatially Adaptive Statistical Modeling of Wavelet Image Coefficients and Its Application to Denoising," IEEE International Conference on Acoustics, Speech, and Signal Processing, Vol. 6, 1999, pp. 3253 - 3256.

[10] M. K. Mihcak, I. Kozintsev, K. Ramchandran and P. Moulin, "Low-complexity image denoising based on statistical modeling of wavelet coefficients," IEEE Signal Processing Letters, Vol. 6, No. 12, 1999, pp. 300-303.

[11] C. Burrus, R. Gopinath, and H. Guo, Introduction to Wavelets and Wavelet Transforms: A Primer, Prentice Hall, 1998

[12] R. M. Rao and A. S. Bopardikar, Wavelet Transforms: Introduction To Theory And Application, Addison Wesly, 2001, pp. 1-126.

[13] Y. Meyer, Wavelets and operators, Cambridge University Press, 1992.

[14] M. Vetterli and J. Kovacevic, Wavelets and Subband Coding, Englewood Cliffs, New Jersey, Prentice Hall, 1995.

[15] H. Om and M. Biswas, "A Generalized Image Denoising Method Using Neighbouring Wavelet Coefficients," Signal Image and Video Processing (Springer), under review, 2012 
[16] R. K. Rai, J. Asnani and T. R. Sontakke, "Review of Shrinkage Techniques for Image Denoising," International Journal of Computer Applications, Vol. 42, No. 19, 2012, pp. 13-16.

[17] A. Kalita, Md. S. Hossain and K. K. Sarma, "A New Approach to Image Denoising based on Wiener-LMMSE Scheme," International Journal of Computer Applications, Vol. 45, No. 22, 2012, pp. 41-47.
[18] H. Om and M. Biswas, "An Improved Image Denoising Method based on Wavelet Thresholding," Journal of Signal and Information Processing (USA), Vol. 3, No. 1, 2012, pp.109-116.

[19] B. C. Rao and M. M. Latha, "Selective Neighbouring Wavelet Coefficients Approach For Image Denoising," International Journal of Computer Science and Communication, Vol. 2, No. 1, 2011, pp. 73- 77.

Table 1. Denoising numerical results (PSNR in db) for Lena, Mandrill, Barbara, Goldhill, and Cameraman with window sizes $3 \times 3,5 \times 5$, and $7 \times 7$ for Weiner Filter, NeighShrink, and LAWML methods with noise levels: 10, 20, 30, 50, 75, and 100 and $n=1,2 \& 4$

\begin{tabular}{|c|c|c|c|c|c|c|c|c|c|c|c|c|c|c|c|c|c|c|c|}
\hline \multirow{4}{*}{ 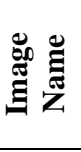 } & \multirow{4}{*}{$\begin{array}{ll}\frac{0}{0} \\
\frac{0}{0} \\
\frac{0}{0} \\
z\end{array}$} & \multicolumn{6}{|c|}{ Weiner Filter NeighShrink } & \multicolumn{3}{|c|}{ LAWML } & \multicolumn{9}{|c|}{ Proposed Method with value of $n$ value } \\
\hline & & \multicolumn{18}{|c|}{ PSNR } \\
\hline & & \multirow[t]{2}{*}{$3 \times 3$} & \multirow[t]{2}{*}{$5 \times 5$} & \multirow[t]{2}{*}{$7 \times 7$} & \multirow[t]{2}{*}{$3 \times 3$} & \multirow[t]{2}{*}{$5 \times 5$} & \multirow[t]{2}{*}{$7 \times 7$} & \multirow[t]{2}{*}{$3 \times 3$} & \multirow[t]{2}{*}{$5 \times 5$} & \multirow[t]{2}{*}{$7 \times 7$} & $3 \times 3$ & $5 \times 5$ & $7 \times 7$ & $3 \times 3$ & $5 \times 5$ & $7 \times 7$ & $3 \times 3$ & $5 \times 5$ & $7 \times 7$ \\
\hline & & & & & & & & & & & \multicolumn{3}{|c|}{$n=1$} & \multicolumn{3}{|c|}{$n=2$} & \multicolumn{3}{|c|}{$n=4$} \\
\hline \multirow{6}{*}{ 苂 } & 10 & 33.55 & 32.67 & 31.37 & 33.29 & 34.22 & 31.37 & 33.48 & 34.14 & 34.46 & 33.59 & 33.99 & 34.23 & 33.62 & 34.01 & 34.25 & 33.64 & 34.03 & 34.26 \\
\hline & 20 & 28.99 & 30.01 & 29.33 & 28.59 & 30.40 & 30.97 & 28.77 & 29.52 & 29.99 & 29.70 & 29.70 & 29.82 & 29.76 & 29.77 & 29.86 & 29.78 & 29.83 & 29.89 \\
\hline & 30 & 25.70 & 27.83 & 27.74 & 26.09 & 27.58 & 28.70 & 26.13 & 26.71 & 27.08 & 28.23 & 27.74 & 27.51 & 28.29 & 27.88 & 27.60 & 28.29 & 27.98 & 27.66 \\
\hline & 50 & 21.40 & 24.48 & 25.37 & 23.47 & 24.59 & 25.43 & 23.64 & 23.83 & 24.02 & 26.92 & 25.92 & 25.26 & 26.92 & 26.27 & 25.48 & 26.83 & 26.50 & 25.65 \\
\hline & 75 & 7.95 & 21.51 & 23.09 & 22.51 & 22.93 & 23.52 & 22.60 & 22.62 & 22.66 & 25.59 & 24.29 & 23.12 & 25.44 & 24.89 & 23.53 & 25.23 & 25.23 & 23.85 \\
\hline & 100 & 15.49 & 19.26 & 21.23 & 22.06 & 22.09 & 22.49 & 22.09 & 22.07 & 22.06 & 24.45 & 23.10 & 21.47 & 24.20 & 23.89 & 22.06 & 23.98 & 24.27 & 22.52 \\
\hline \multirow{6}{*}{ 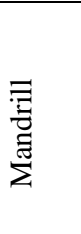 } & 10 & 0 & 24.50 & 23.57 & 12 & 29.86 & 28.12 & .97 & 29.31 & 30.02 & 06 & 29.32 & 30.00 & 28.06 & 29.32 & 30.00 & 28.06 & 129.32 & 30.00 \\
\hline & 20 & 79 & 23.54 & 22.83 & 22.12 & 24.60 & 25.32 & 22.08 & 23.12 & 23.99 & 22.58 & 23.35 & 24.07 & 22.58 & 23.35 & 24.08 & 22.58 & 23.36 & 24.08 \\
\hline & 30 & 23.08 & 22.60 & 22.09 & 20.16 & 21.47 & 22.86 & 20.18 & 20.60 & 21.06 & 21.18 & 21.28 & 21.48 & 21.17 & 21.29 & 21.49 & 21.16 & 21.31 & 21.50 \\
\hline & 50 & 20.13 & 21.00 & 20.95 & 19.37 & 19.60 & 20.07 & 19.38 & 19.44 & 19.51 & 20.55 & 20.41 & 20.27 & 20.50 & 20.48 & 20.32 & 20.44 & 20.52 & 20.36 \\
\hline & 75 & \begin{tabular}{|l}
17.30 \\
\end{tabular} & 19.29 & 19.83 & 19.14 & 19.20 & 19.33 & 19.15 & \begin{tabular}{|l|l}
19.14 \\
\end{tabular} & 19.15 & 20.05 & 19.82 & 19.43 & 19.94 & 19.99 & 19.58 & 19.84 & 20.07 & 19.69 \\
\hline & 100 & 15.10 & \begin{tabular}{|l|l}
17.77 \\
\end{tabular} & $\mid 18.80$ & 19.04 & 19.04 & 19.05 & 19.04 & 19.04 & 19.04 & 19.63 & 19.30 & 18.62 & 19.51 & 19.58 & 18.89 & 19.43 & 19.69 & 19.09 \\
\hline \multirow{6}{*}{ 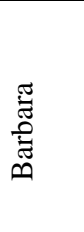 } & 10 & & 28.03 & 27.17 & .28 & 32.51 & 30.58 & 31.35 & 32.36 & 32.73 & 31.48 & 32.31 & 32.64 & 31.49 & 32.32 & 32.65 & 31.50 & 32.33 & 32.65 \\
\hline & 20 & 26.82 & 26.24 & 25.81 & 25.33 & 27.71 & 28.52 & 25.35 & 26.48 & 27.20 & 25.86 & 26.72 & 27.25 & 25.88 & 26.76 & 27.27 & 25.88 & 26.78 & 27.29 \\
\hline & 30 & 24.29 & 24.71 & 24.63 & 22.58 & 24.48 & 25.92 & 22.66 & 23.33 & 23.92 & 23.61 & 23.91 & 24.23 & 23.62 & 23.96 & 24.26 & 23.62 & 23.99 & 24.29 \\
\hline & 50 & 20.66 & 22.38 & 22.95 & 21.07 & 21.74 & 22.36 & 21.13 & 21.30 & 21.47 & 22.89 & 22.50 & 22.21 & 22.87 & 22.64 & 22.31 & 22.82 & 22.73 & 22.38 \\
\hline & 75 & 7.55 & 20.18 & 21.36 & 20.39 & 20.53 & 21.11 & 20.44 & 20.45 & 20.46 & 22.26 & 21.66 & 21.01 & 22.16 & 21.96 & 21.25 & 22.05 & 22.12 & 21.42 \\
\hline & 100 & 15.25 & 18.38 & 19.99 & 20.18 & 20.22 & 20.32 & 20.18 & 20.19 & 20.20 & 21.64 & 20.95 & 19.91 & 21.48 & 21.40 & 20.31 & 21.33 & 21.60 & 20.60 \\
\hline \multirow{6}{*}{$\begin{array}{l}\bar{\Xi} \\
\overline{0} \\
0 \\
0\end{array}$} & 10 & 31.78 & 30.30 & 28.97 & 30.79 & 32.22 & 30.41 & 30.86 & 31.77 & 32.35 & 31.06 & 31.77 & 32.28 & 31.07 & 31.78 & 32.29 & 31.07 & 31.79 & 32.29 \\
\hline & 20 & 28.26 & 28.57 & 27.77 & 26.73 & 28.21 & 29.08 & 26.72 & 27.31 & 27.77 & 27.79 & 27.83 & 27.95 & 27.81 & 27.87 & 27.97 & 27.81 & 27.89 & 27.99 \\
\hline & 30 & 25.35 & 26.92 & 26.69 & 24.80 & 26.11 & 26.99 & 24.85 & 25.30 & 25.66 & 26.79 & 26.52 & 26.37 & 26.80 & 26.61 & 26.43 & 26.76 & 26.67 & 26.47 \\
\hline & 50 & 21.27 & 24.06 & 24.81 & 23.43 & 23.91 & 24.63 & 23.45 & 23.61 & 23.75 & 25.73 & 25.09 & 24.59 & 25.67 & 25.36 & 24.77 & 25.55 & 25.52 & 24.90 \\
\hline & 75 & 17.89 & 21.29 & 22.78 & 22.67 & 22.99 & 23.32 & 22.72 & 22.77 & 22.83 & 24.65 & 23.71 & 22.72 & 24.50 & 24.22 & 23.09 & 24.32 & 24.49 & 23.37 \\
\hline & 100 & 15.45 & \begin{tabular}{|l}
19.12 \\
\end{tabular} & 21.03 & 22.20 & 22.26 & 22.44 & 22.23 & 22.23 & 22.21 & 23.76 & 22.68 & 21.21 & 23.57 & 23.38 & 21.77 & 23.41 & 23.71 & 22.19 \\
\hline & 10 & 32.77 & \begin{tabular}{|l|l}
31.47 \\
\end{tabular} & 30.39 & 32.72 & 33.87 & 31.23 & 32.88 & 33.77 & 34.05 & 32.95 & 33.61 & 33.83 & 32.98 & 33.63 & 33.84 & 33.00 & 33.65 & 33.86 \\
\hline & 20 & 28.73 & 29.14 & 28.49 & 26.96 & 28.81 & 29.71 & 27.05 & 27.76 & 28.22 & 27.58 & 27.84 & 28.08 & 27.62 & 27.89 & 28.11 & 27.63 & 27.93 & 28.13 \\
\hline స్త్ర & 30 & 25.52 & 27.11 & 26.83 & 24.59 & 26.08 & 27.05 & 24.73 & 25.22 & 25.52 & 25.88 & 25.73 & 25.67 & 25.92 & 25.82 & 25.72 & 25.93 & 25.89 & 25.76 \\
\hline 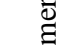 & 50 & 21.23 & 23.91 & 24.40 & 21.89 & 22.82 & 24.13 & 21.88 & 22.33 & 22.53 & 24.36 & 23.86 & 23.44 & 24.37 & 24.07 & 23.58 & 24.32 & 24.20 & 23.68 \\
\hline Uే & 75 & 17.82 & 21.06 & 22.21 & 20.37 & 20.72 & 21.74 & 20.32 & 20.50 & 20.69 & 23.34 & 22.52 & 21.71 & 23.24 & 22.90 & 21.99 & 23.11 & 23.10 & 22.19 \\
\hline & 100 & 15.39 & 18.93 & 20.52 & \begin{tabular}{|l}
19.87 \\
\end{tabular} & 19.99 & 20.63 & 19.86 & 19.90 & 19.98 & 22.51 & 21.63 & 20.41 & 22.31 & 22.16 & 20.85 & 22.13 & 22.41 & 21.18 \\
\hline
\end{tabular}

